Oslo TP 17-93

ZTF - 93/11

\title{
New contribution to anomalous radiative $K^{0}$-decays
}

\author{
J. O. Eeg' \\ Dept. of Physics, University of Oslo, N-0316 Oslo, Norway \\ I. Picek? \\ Fizički odjel, University of Zagreb, POB 162, 41000 Zagreb, Croatia
}

\begin{abstract}
We present a new contribution to the $K_{L} \rightarrow \gamma \gamma$ amplitude, which is $\mathcal{O}\left(p^{4}\right)$ within the counting rules of chiral perturbation theory. This direct (non-pole) amplitude, obtained from short-distance $s \rightarrow d \gamma$ quark diagrams of order $e G_{F} \alpha_{s} / \pi$, and similar $s \rightarrow d \gamma \gamma$ diagrams, can account for about half of the experimental amplitude. Closely following the description of the $\pi^{0} \rightarrow \gamma \gamma$ and $K_{L} \rightarrow \gamma \gamma$ processes in the variants of the same low-energy QCD, we find that both of these processes are anomalous in the same sense. Then, by the anomaly-matching principle, we arrive from the chiral-quark to the bosonic counterparts for both of these processes. In this way we add the $K_{L} \rightarrow \gamma \gamma$ decay to the existing list of anomalous radiative prosesses.
\end{abstract}

\footnotetext{
${ }^{1}$ e-mail : eeg@vuoep1.uio.no

${ }^{2}$ e-mail : picek@phy.hr
} 


\section{Introduction}

Radiative kaon decays appear to be suitable for uncovering the subtleties of overbridging the quark and the hadronic worlds. This procedure conventionally derives the effective bosonic operators of the Chiral Perturbation Theory $(\chi \mathrm{PT})$, starting from four-quark operators at the free-quark level [1].

In this paper we recall our recent experience in transferring a two-quark operator at quark level to a contribution to the $\overline{K^{0}} \rightarrow \gamma \gamma$ amplitude [2]. In an earlier paper [3] we pointed out the existence of pure electroweak shortdistance $s \rightarrow d \gamma \gamma$ loop diagrams that gave an important contribution to the CP-violating $\overline{K^{0}} \rightarrow \gamma \gamma$ amplitude. The significance of the two-quark $s \rightarrow d \gamma \gamma$ operator was due to the non-efficient GIM cancellation for a heavy top-quark in the relevant loop diagrams. Completing these irreducible diagrams [3] with the reducible ones gave large cancellations for free on-shell $s, d$ quarks [4]. Using an effective low-energy QCD advocated by many authors [5], we transformed the short distance quark result into the meson-"world" amplitude [2], for which these cancellations were lifted. Our new $\overline{K^{0}} \rightarrow \gamma \gamma$ amplitude obtained in this way is $\mathcal{O}\left(p^{4}\right)$ within the counting rules of $\chi \mathrm{PT}$. However, to our knowledge, there are no such direct (non-pole) $\chi P T$ terms $\mathcal{O}\left(p^{4}\right)$ in the literature.

In the present paper we dwell on the appearance of this $\overline{K^{0}} \rightarrow \gamma \gamma$ amplitude at the hadronic level. Thereby we rely on the close relation which we demonstrate to exist between the $\pi^{0} \rightarrow \gamma \gamma$ and $K_{L} \rightarrow \gamma \gamma$ amplitudes. Whereas the former is known to be governed by the anomalous Wess-ZuminoWitten (WZW) [6] term, which is $\mathcal{O}\left(p^{4}\right)$, the existing locally chiral-invariant flavour-changing contributions of $\mathcal{O}\left(p^{4}\right)$ considered in [7, 8, 9] correspond to four-quark operators, and do not contribute to $\overline{K^{0}} \rightarrow \gamma \gamma$. The anomalous terms presented by these authors [0, 8, 9], termed the direct anomalous ones, can be constructed from a combination of the anomalous current obtained from the WZW term (responsible for $\pi^{0} \rightarrow \gamma \gamma$ ) and the current obtained from the normal term $\mathcal{O}\left(p^{2}\right)$. The reducible anomalous pole diagrams, including directly the WZW term for $\pi^{0}, \eta \rightarrow 2 \gamma$, vanish in the $\mathrm{SU}(3)$ limit where the Gell-Mann-Okubo mass formula is used. The remaining reducible anomalous contributions to $\overline{K^{0}} \rightarrow \gamma \gamma$ come from $\mathcal{O}\left(p^{6}\right)$ corrections to the Gell-Mann-Okubo mass formula.

The purpose of this paper is twofold: First, we present a CP-conserving counterpart of ref. [3]. Such a CP-conserving $\overline{K^{0}} \rightarrow \gamma \gamma$ amplitude, suffering from the GIM cancellation in the pure electroweak case, has to be of order $e^{2} G_{F} \alpha_{s} / \pi$, as shown in some of the early literature on this process[10]. Second, we demonstrate the anomalous nature of this process (in the low-energy QCD at hand) and discuss how our $\mathcal{O}\left(p^{4}\right), \overline{K^{0}} \rightarrow \gamma \gamma$ amplitude, corresponding to two-quark operators, can be included among $\Delta S=1$ chiral lagrangian terms. 


\section{The quark evaluation of the $K_{L} \rightarrow \gamma \gamma$ amplitude}

In previous papers we studied electroweak quark loops for $s \rightarrow d \gamma$ and $s \rightarrow d \gamma \gamma$ [3, 团, where the parts related by Ward identities can be combined into the effective lagrangian

$$
\mathcal{L}(s \rightarrow d)_{\gamma}=B \epsilon^{\mu \nu \lambda \rho} F_{\mu \nu}\left(\overline{d_{L}} i \stackrel{\leftrightarrow}{D_{\lambda}} \gamma_{\rho} s_{L}\right) .
$$

The quantity $B$ contains short distance loop effects from scales above the chiral symmetry breaking scale $\sim 1 \mathrm{GeV} . F_{\mu \nu}$ is the electromagnetic field

tensor. The covariant derivative $\stackrel{\leftrightarrow}{D_{\lambda}}$, containing another electromagnetic field $A_{\lambda}$, acts on left-handed $s$ - and $d$ - quark fields. Having obtained $\mathcal{L}(s \rightarrow d)_{\gamma}$, one often removes the covariant derivative by using the equations of motion. Then one obtains the well-known magnetic moment term[11]. In other words, one can split $\mathcal{L}(s \rightarrow d)_{\gamma}$ in two terms (eqs. (9) and (10) of ref.[狽), the one which vanishes at the free-quark mass-shell and the other (the magnetic moment term of $\mathcal{O}\left(p^{6}\right)$ in $\left.\chi P T\right)$ that vanishes in the limit $m_{s, d} \rightarrow 0$.

The quantity $B$ is of order $e \cdot G_{F}$ and, for convenience, let us introduce a dimensionless quantity $\hat{B}$ :

$$
B=\frac{G_{F}}{\sqrt{2}} \frac{e}{4 \pi^{2}} \hat{B}
$$

Furthermore, $\hat{B}$ can be split in two parts with different KM-factors $\lambda_{q}=$ $V_{q s} V_{q d}^{*}(q=u, c, t)$,

$$
\hat{B}=\lambda_{u} \hat{B}_{u}+\lambda_{t} \hat{B}_{t}
$$

The first term is purely CP-conserving (CP-even), whereas the second contributes to the CP-violating effects determined by $\operatorname{Im}\left(\lambda_{t}\right)$.

In the present theoretical approach, the $\overline{K^{0}} \rightarrow \gamma \gamma$ amplitude is calculated within a low-energy QCD model from quark-level diagrams. This low-energy QCD [5] is obtained by adding a new term to ordinary QCD:

$$
\mathcal{L}_{\chi}=-M\left(\overline{q_{R}} U q_{L}+\overline{q_{L}} U^{\dagger} q_{R}\right)
$$

where $\bar{q}=(\bar{u}, \bar{d}, \bar{s})$ and the 3 by 3 matrix $U \equiv \exp \left(\frac{2 i}{f} \Pi\right)$ contains the pseudoscalar octet mesons $\Pi=\sum_{a} \pi^{a} \lambda^{a} / 2(a=1, . ., 8)$, and $f$ can be identified with the pion decay constant, $f=f_{\pi}=(92.4 \pm 0.2) \mathrm{MeV}\left(=f_{K}\right.$, in the chiral limit). The resulting field theory with pseudoscalar quark-meson couplings and quark loops reproduces the well-known $\pi^{0} \rightarrow \gamma \gamma$ amplitude.

The $K^{0}$-decay amplitude we study here has the form

$$
A\left(\overline{K^{0}} \rightarrow \gamma \gamma\right)=\sqrt{2} \mathcal{R} \epsilon_{\mu \nu \rho \sigma} \epsilon_{1}^{\mu} \epsilon_{2}^{\nu} k_{1}^{\rho} k_{2}^{\sigma},
$$


where the quantity $\mathcal{R}$ can be split in two parts as in (3). The results which we presented in ref. [2] imply that even in the chiral limit $m_{s} \rightarrow 0$ there is a non-zero contribution to $\mathcal{R}$ from $\mathcal{L}(s \rightarrow d)_{\gamma}$ of order $\mathcal{O}\left(p^{4}\right)$,

$$
\mathcal{R}=-4 h_{B} e_{D} B
$$

Here $e_{D}=e Q_{D}=-e / 3$ is the electric charge of the down $(d, s)$ quarks. The quantity $h_{B}$ is the hadronic matrix element of the quark operator within the parenthesis in (1) calculated within an effective low-energy QCD described

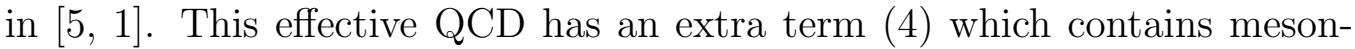
quark couplings proportional to the constituent quark mass $M \sim 300 \mathrm{MeV}$ and to inverse powers of $f_{\pi}$. The chiral symmetry-breaking scale $\Lambda_{\chi} \sim 0.7$ to $1 \mathrm{GeV}$ is the natural UV cut-off. Potentially UV-divergent integrals $\sim$ $\Lambda_{\chi}^{2}$ and $\log \left(\Lambda_{\chi}\right)$ are absorbed in the quark condensate and the physical $f_{\pi}$, respectively. The result of the calculation is [2]

$$
h_{B}=-\frac{N_{c} M^{2} \Delta_{L D}}{4 \pi^{2} f_{\pi}}
$$

The quantity $\Delta_{L D}$ is a dimensionless function of the ratio $M^{2} / \Lambda_{\chi}^{2}$. Using dimensional regularization, corresponding to the formal limit $\Lambda_{\chi} \rightarrow \infty$, we find $\Delta_{L D}=-2$. The quantity $h_{B}$ in (6) is the sum of two terms [2]: The one with the hadronic matrix element $\sim f_{K}$ obtained for $i D_{\sigma} \rightarrow e_{D} A_{\sigma}$ in (1) corresponding to irreducible electroweak diagrams for $s \rightarrow d \gamma \gamma$; the other term, having a hadronic matrix element $\sim f_{L D}=f_{K}-h_{B}$, is obtained when $D_{\sigma} \rightarrow \partial_{\sigma}$ in (1), and corresponds to an irredicible diagram for $s \rightarrow d \gamma$ with a photon on an external $(s-$ or $d-$ ) quark, i.e. to a reducible diagram for $s \rightarrow d \gamma \gamma$. It should be noted that the total contribution $f_{K}-f_{L D}=h_{B} \rightarrow 0$ in the limit $f_{\pi} \rightarrow \infty$ when the quark-meson interactions are switched off, corresponding to the free-quark case. There are also other operators, e.g. the non-diagonal $s \rightarrow d$ self-energy, but these turn out to have small coefficients and do not contribute significantly to $K \rightarrow 2 \gamma$.

In the following we concentrate on the CP-conserving case. In order to explain $K_{L} \rightarrow \gamma \gamma$ by $\mathcal{L}(s \rightarrow d)_{\gamma}$ alone, a numerical value $\hat{B}_{u} \simeq 1.5$ is needed and pure electroweak diagrams such as in [3] cannot account for this in the CP-conserving case. However, some QCD-induced contributions exist. A typical contribution, which has already been known for some time [11], corresponds to

$$
\hat{B}_{u}=2 Q_{U} \frac{\alpha_{s}}{\pi} \ln \frac{m_{c}^{2}}{\mu^{2}}
$$

where $Q_{U}=2 / 3$ is the charge of up (u,c) quarks, and $\mu$ is the renormalization scale, typically of order $1 \mathrm{GeV}$. Taking this expression at face value gives $\hat{B}_{u} \simeq 0.3$ for $\mu=\Lambda_{\chi}$ and $\hat{B}_{u} \simeq 1.2$ for $\mu=M$. However, the physical result 
corresponding to the leading logarithmic perturbative result (8) is difficult to estimate reliably because the charm scale is rather close to the confinement and the chiral-symmetry breaking scales (so that next-to-leading-log terms might be important). Taking into account that $\mu$ corresponds to the $s, d$ quark momenta in (1), subsequently being the loop momenta in the effective low-energy QCD [5], we find that $\hat{B}_{u} \simeq 0.6$ to 1 is a reasonable range for the value based on (8). In previous papers [12, 13] we studied "penguininduced" two-loop contributions to $s \rightarrow d \gamma, d \gamma \gamma$ and $b \rightarrow s \gamma$, where we focused on the $(c, t)$ quarks in the loop. There will also be corresponding CP-conserving terms $\sim \lambda_{u}$ for $s \rightarrow d \gamma, d \gamma \gamma$ with $(u, c)$ quarks in the loops. These will, however, give too small a contribution to $\hat{B}_{u}$. There will also be contributions of the non-diagonal self-energy type [2] which are non-zero, but small.

Taking into account the uncertainty of perturbative calculations on the 1 GeV scale, QCD-induced contributions will probably reproduce roughly half of the $K_{L} \rightarrow \gamma \gamma$ decay amplitude. These SD contributions require additional next-to-leading corrections to (8) in order to reach a more precise conlusion. In addition, there might be a significant long distance contribution from improper cancellation of pole diagrams.

\section{Anomalous nature of the $K_{L} \rightarrow \gamma \gamma$ amplitude}

In order to establish the anomalous nature of the $K_{L} \rightarrow \gamma \gamma$ process, we perform a comparative study of $K_{L} \rightarrow \gamma \gamma$ and $\pi^{0} \rightarrow \gamma \gamma$ processes. Both of them are governed by an effective interaction of the form

$$
\mathcal{L}_{X}=\alpha C_{X} \epsilon_{\mu \nu \rho \sigma} F^{\mu \nu} F^{\rho \sigma} \Phi_{X}
$$

for $X=\pi^{0}$ or $X=K_{2}$. The $\pi^{0} \rightarrow \gamma \gamma$ rate is reproduced by

$$
C_{\pi^{0}}=\frac{N_{c}}{24 \pi f_{\pi}}=4.3 \times 10^{-4} \mathrm{MeV}^{-1} .
$$

The rate for $K_{2} \rightarrow \gamma \gamma$ similarly determines the phenomenological coupling

$$
\left|C_{K_{2}}\right|=5.9 \times 10^{-11} \mathrm{MeV}^{-1} .
$$

Notably, the adopted low-energy QCD accounts for the full $C_{\pi^{0}}$ amplitude, whereas the calculation in the previous section shows that it accounts for roughly a half of the $\left|C_{K_{2}}\right|$. This refers to the "unrotated" $(U)$ version of low-energy QCD [5].

However, the term $\mathcal{L}_{\chi}$ in (4) can be transformed into a pure mass term $-M \overline{\mathcal{Q}} \mathcal{Q}$ for rotated "constituent quark" fields $\mathcal{Q}_{L, R}$ :

$$
q_{L} \rightarrow \mathcal{Q}_{L}=\xi q_{L} ; \quad q_{R} \rightarrow \mathcal{Q}_{R}=\xi^{\dagger} q_{R} ; \xi \cdot \xi=U
$$


Then the meson-quark couplings in this "rotated" $(R)$ picture are transformed into the kinetic (Dirac) part of the "constituent quark" lagrangian. These interactions can be described in terms of vector and axial vector fields coupled to constituent quark fields $\mathcal{Q}=\mathcal{Q}_{R}+\mathcal{Q}_{L}$ :

$$
\begin{array}{r}
\mathcal{L}_{\text {int }}=\overline{\mathcal{Q}}\left[\gamma^{\mu} \mathcal{V}_{\mu}+\gamma^{\mu} \gamma_{5} \mathcal{A}_{\mu}\right] \mathcal{Q} \\
\mathcal{V}_{\mu}=\left(R_{\mu}+L_{\mu}\right) / 2 ; \mathcal{A}_{\mu}=\left(R_{\mu}-L_{\mu}\right) / 2 ; \\
L_{\mu}=\xi\left(i \partial_{\mu} \xi^{\dagger}\right)+\xi l_{\mu} \xi^{\dagger} ; R_{\mu}=\xi^{\dagger}\left(i \partial_{\mu} \xi\right)+\xi^{\dagger} r_{\mu} \xi
\end{array}
$$

Here $l_{\mu}$ and $r_{\mu}$ are the external fields containing the photon (eventually also the $\mathrm{W}$ field). A calculation of the $\pi^{0} \rightarrow \gamma \gamma$ amplitude in the rotated $(R)$ picture gives a zero result (for infinite cut-off, i.e. by dimensional regularization). We have found by explicit calculation that this is also the case for the $\overline{K^{0}} \rightarrow \gamma \gamma$ amplitude!

For the $\pi^{0} \rightarrow \gamma \gamma$ decay, the interpretation is unambiguous. In the rotated basis, where pions have proper derivative Goldstone-couplings, the compensating WZW term ensures the anomaly matching. The unrotated-quarktriangle evaluation finds a counterpart in the anomalous WZW part of the chiral lagrangian. The explicit diagrammatic evaluation complies with the more general functional derivation of the Wess-Zumino-Witten (WZW) term [6], which is contained in a Jacobian of the quark field rotation in eq. (10).

The results (non-zero in the $U$-basis, zero in the $R$-basis) in evaluating the $\overline{K^{0}} \rightarrow \gamma \gamma$ amplitude from quark-level diagrams within low-energy QCD, motivates us to attribute a similar anomalous nature to this process! By employing the anomaly-matching principle, we argue for the existence of the related bosonic lagrangian term corresponding to the WZW term.

In this connection we should stress an important point, namely that the wanted bosonic lagrangian is based on the underlying $s \rightarrow d \gamma \gamma$ transition that is not of the current-current form explored in the literature [7, 8, 9]. In the chiral-invariant version, the quark operator (1) can be rewritten in a form where its $\left(8_{L}, 1_{R}\right)$ structure is manifest. For convenience, we also perform the Dirac algebra in (1) in order to bring it to the form containing $\sigma_{\mu \nu}$ instead of the Levy-Civita tensor (which obscures the dimensional regularization):

$$
\mathcal{L}(s \rightarrow d)_{\gamma}=(-3) B \overline{q_{L}} \lambda_{+}\left[i \stackrel{\leftarrow}{D} \cdot \gamma \sigma^{\mu \nu} F_{\mu \nu}^{L}+\sigma^{\mu \nu} F_{\mu \nu}^{L} i \gamma \cdot \vec{D}\right] q_{L}
$$

In this expression, $D_{\sigma} q_{L}=\left(\partial_{\sigma}-i l_{\sigma}\right) q_{L}, \bar{\psi} \stackrel{\overleftarrow{\partial_{\sigma}}}{=}-\partial_{\sigma}(\bar{\psi})$, the field-strength tensor $F_{\mu \nu}^{L}=\partial_{\mu} l_{\nu}-\partial_{\nu} l_{\mu}-i\left[l_{\mu}, l_{\nu}\right]$, and the factor $(-3)$ compensates the charge $Q$ contained in the field $l_{\mu}$. For the purely electromagnetic gauging, this field is

$$
l_{\mu}=r_{\mu} \equiv e A_{\mu} Q ; \quad Q=\frac{1}{3} \operatorname{diag}(2,-1,-1)
$$


The Gell-Mann matrices $\lambda_{ \pm}=\left(\lambda_{6} \pm i \lambda_{7}\right) / 2$ project $\Delta S= \pm 1$ transitions out of the quark fields $\bar{q}_{L}=\left(\bar{u}_{L}, \bar{d}_{L}, \bar{s}_{L}\right)$. Note that in (12) the covariant derivative contains only the left-handed field $l_{\sigma}$, and that the operator transforms as $\left(8_{L}, 1_{R}\right)$ under the local chiral $S U(3)_{L} \times S U(3)_{R}$ symmetry. Obviously, because of the anomalous nature of $K^{0} \rightarrow 2 \gamma$, we have established, the local chiral invariance should be lost, and we expect that our amplitude is in some way related to the WZW term.

In ref. [2] it is shown that the term $\mathcal{L}(s \rightarrow d)_{\gamma}$ can be transformed away into the kinetic QCD term. However, compensating interactions are then appearing in the "mass term" (4), and can be described by a new lagrangian, which has an appealing form after performing the rotation (10) (which simultaneously gives (11)) :

$$
\Delta \mathcal{L}_{\chi}^{\Delta S=1}=(-3) B M \overline{\mathcal{Q}} \sigma_{\mu \nu} T_{L(+)}^{\mu \nu} \mathcal{Q},
$$

where

$$
T_{L(+)}^{\mu \nu}=\xi \lambda_{+} F_{\mu \nu}^{L} \xi^{\dagger}
$$

encapsulates the flavour change in the photon-emission vertex. Note that also from $\Delta \mathcal{L}_{\chi}^{\Delta S=1}$ we have found by explicit calculation, using dimensional regularization, that $\Delta_{L D}=-2$ and $\Delta_{L D}=0$ in the $U$ - and $R$ - pictures respectively (see eq.(7)), thus confirming the anomalous charachter of $K^{0} \rightarrow$ $2 \gamma$. In the next section we will show how the expressions (11) and (13) enable us to find the general structure of the hadronic $\mathcal{O}\left(p^{4}\right)$ term responsible for the $K^{0} \rightarrow 2 \gamma$ process.

\section{Bosonization}

Our final task refers to " bosonizing" the operator contributing to $K^{0} \rightarrow$ $2 \gamma$, induced by (1) or (12) or by "the intermediate" expression, which still includes the Levy-Civita tensor:

$$
\mathcal{L}(s \rightarrow d)_{\gamma}=(-3) B \epsilon^{\mu \nu \sigma \rho} \overline{q_{L}} \lambda_{+}\left(i \stackrel{\overleftarrow{D_{\sigma}}}{F_{\mu \nu}^{L}}+F_{\mu \nu}^{L} i \overrightarrow{D_{\sigma}}\right) \gamma_{\rho} q_{L}
$$

A direct bosonization of this operator is difficult because of the covariant derivative sitting between the quark fields. However, we observe that by keeping only the $l_{\mu}$ part of the covariant derivative (corresponding to purely electromagnetic gauging), we obtain the promising structure

$$
\epsilon^{\mu \nu \sigma \rho}(\text { quark current })_{\rho} \times l_{\sigma} \times F_{\mu \nu}^{L} .
$$

The bosonized version of the quark current is $\Sigma_{\rho}^{L}=U^{\dagger} \partial_{\rho} U$, the object which can be recognized as a building block of the WZW terms responsible for radiative decays (-see eq.(19) below). 
An elegant way to bosonize quark operators, the heat kernel method, is demonstrated in [1]. In the present paper we are, however, sticking to quark loop diagrams which give less information on the bosonized counterpart of $\mathcal{L}(s \rightarrow d)_{\gamma}$ in eqs.(1), (12) and (15). Indeed, in the $U$ - picture loop calculation, the momenta which correspond to derivatives on fields have to be pulled out of the quark propagators of the loop diagram, and we loose structures like (16). However, this is not so in the $R$-picture, where the vector and axial vector fields in eq.(11) couple in two of the vertices of the triangle loop diagram for $\overline{K^{0}} \rightarrow 2 \gamma$, and the interaction given by (13) acts in the third vertex. Then, for $\mathcal{O}\left(p^{4}\right)$ terms, no momenta have to be pulled out of the quark propagators, and we can deduce the typical anomalous contribution:

$$
\mathcal{L}_{A n}^{\Delta S=1} \sim \frac{M^{2} N_{c}}{4 \pi^{2} f_{\pi}} B \epsilon^{\mu \nu \alpha \beta} \operatorname{Tr}\left[T_{L}^{(+)}{ }_{\mu \nu} \mathcal{V}_{\alpha} \mathcal{A}_{\beta}\right]
$$

There will also be other terms with the structure $\mathcal{V} \mathcal{A}$ replaced by $\mathcal{A} \mathcal{V}, \mathcal{V} \mathcal{V}$, or $\mathcal{A} \mathcal{A}$, which will appear in the total lagrangian with different coefficients. These coefficients have to be determined in the $U$-picture, by considering quark loop diagrams for processes to which (17) contributes. It is known [5] that $\mathcal{A}_{\mu}$ is locally invariant, whereas the vector field $\mathcal{V}_{\mu}$ is not. Therefore, the terms involving the vector field manifestly break the local chiral invariance in a transparent way. We will discuss expression (17) in full detail elsewhere. Here we explicate this formula for the $K^{0} \rightarrow 2 \gamma$ process by comparing it to a more familiar WZW term: Operators like (17) might be obtained by appropriate $\lambda_{+}$insertions in the WZW action.

Some comments on the ordinary Wess-Zumino-Witten (WZW) term [6] are in order. Apart from the hadronic term (relevant to $K^{0} \overline{K^{0}} \rightarrow 3 \pi$ ) there is a part relevant to the Goldstone-to-two-photon radiative decays under consideration:

$$
\mathcal{L}_{W Z W}=-\frac{i N_{c}}{48 \pi^{2}} \epsilon^{\mu \nu \alpha \beta} \operatorname{Tr} V_{\mu \nu \alpha \beta}
$$

where

$$
V_{\mu \nu \alpha \beta}=\Sigma_{\mu}^{L} U^{\dagger} \partial_{\nu} r_{\alpha} U l_{\beta}+\Sigma_{\mu}^{L} l_{\nu} \partial_{\alpha} l_{\beta}+\Sigma_{\mu}^{L} \partial_{\nu} l_{\alpha} l_{\beta}+\ldots .-(L \leftrightarrow R) .
$$

The explicit $L \leftrightarrow R$ symmetry interchanging $U$ and $U^{\dagger}$, and $\Sigma_{\mu}^{L}=U^{\dagger} \partial_{\mu} U$ and $\Sigma_{\mu}^{R}=U \partial_{\mu} U^{\dagger}$

$$
U \leftrightarrow U^{\dagger} ; \Sigma_{\mu}^{L} \leftrightarrow \Sigma_{\mu}^{R},
$$

leads to doubling of the "odd $\Pi$ " terms and cancelling of the "even $\Pi$ " terms.

At order $\mathcal{O}\left(p^{4}\right)$ this gives the famous term responsible for $\pi^{0} \rightarrow \gamma \gamma$, which does not involve any unknown coefficients:

$$
\mathcal{L}_{W Z W}^{(4)}=\frac{N_{c} \alpha}{24 \pi f_{\pi}} \epsilon_{\mu \nu \rho \sigma} F^{\mu \nu} F^{\rho \sigma}\left(\pi^{0}+\frac{\eta}{\sqrt{3}}\right)
$$




$$
-\frac{i N_{c} e}{12 \pi^{2} f_{\pi}^{3}} \epsilon_{\mu \nu \rho \sigma} A^{\mu} \partial^{\nu} \pi^{+} \partial^{\rho} \pi^{-} \partial^{\sigma}\left(\pi^{0}+\frac{\eta}{\sqrt{3}}\right)+\ldots . .
$$

Note that the first term in (20) acquires the form written above after applying partial integration to the derivative of the meson field! Before this partial integration the first term had the form

$$
\epsilon_{\mu \nu \rho \sigma} F^{\rho \sigma} A^{\nu} \partial^{\mu}\left(\pi^{0}+\eta / \sqrt{3}\right) .
$$

The two terms in (20) participate in the reducible anomalous (long-distance pole) contributions to $K_{L} \rightarrow 2 \gamma$ and $K_{L} \rightarrow \pi \pi \gamma$, respectively. The other vertex in such pole graphs is given by the weak $K \rightarrow \pi$ transition, displayed below in eq. (23). The fact that the $\pi^{0}$ and $\eta$ pole contributions cancel owing to the Gell-Mann-Okubo mass relation $m_{\eta}^{2}=\frac{1}{3}\left(4 m_{K}^{2}-m_{\pi}^{2}\right)$ arouses interest in the possible existence of important non-pole contributions.

Thus, the question we raise here concerns the possibility of "flavour extension" of expression (19) to the $\Delta S=1$ case, i.e. the possibility of the corresponding expression with $\partial^{\sigma} \pi^{0}$ replaced by $\partial^{\sigma} \overline{K^{0}}$. We observe that the simple substitution

$$
1 \rightarrow \mathcal{G}_{\mathrm{WZW}} \lambda_{+}
$$

in front of $V_{\mu \nu \alpha \beta}$ in (18) provides us with such terms. The lagrangian obtained in this way,

$$
\mathcal{L}_{\mathrm{WZW}}^{\Delta S=1}=-\frac{i N_{c}}{48 \pi^{2}} \mathcal{G}_{\mathrm{WZW}} \epsilon^{\mu \nu \alpha \beta} \operatorname{Tr}\left(\lambda_{+} V_{\mu \nu \alpha \beta}\right),
$$

and the bosonized form $\mathcal{L}_{A n}^{\Delta S=1}$ in (17) both contain the representative terms for $\overline{K^{0}} \rightarrow 2 \gamma$. In general, this prescription is not unique because the $\lambda_{+}$ can be inserted in several ways inside the tensor $V_{\mu \nu \alpha \beta}$ in (19). Thus (21) represents a rather schematic notation: There are different $\mathcal{G}_{\mathrm{WZW}}$ couplings for different insertions of $\lambda_{+}$. A discussion on the different terms of (21) and its relation to (17) will be given elsewhere.

The prescription used to obtain (21) repeats "the Cronin's substitution" leading from the $\mathcal{O}\left(p^{2}\right)$ strong/electromagnetic term

$$
\mathcal{L}_{\text {strong/em }}^{(2)}=\frac{f_{\pi}^{2}}{4} \operatorname{Tr}\left(D^{\mu} U D_{\mu} U^{\dagger}\right)
$$

to the corresponding non-leptonic $\Delta S=1$ term [14]

$$
\mathcal{L}_{\Delta S=1}^{(2)}=g_{8} \operatorname{Tr}\left(\lambda_{+} D^{\mu} U D_{\mu} U^{\dagger}\right)
$$

In close correspondence with the flavour-diagonal terms in (14), our new anomalous term(s) $\mathcal{L}_{\mathrm{WZW}}^{\Delta S=1}$ accounts for the transitions $\overline{K^{0}} \rightarrow \gamma \gamma, \pi \pi \gamma, \pi \pi \gamma \gamma$, 
but not for $\overline{K^{0}} \rightarrow \pi^{0} \gamma \gamma$ :

$$
\begin{array}{r}
\mathcal{L}_{\mathrm{WZW}}^{\Delta S=1}(4)=\mathcal{G}_{\mathrm{WZW}} \\
-\frac{N_{c} \alpha}{24 \pi f_{\pi}} \frac{2 \sqrt{2}}{3} \epsilon_{\mu \nu \rho \sigma} A^{\nu} F^{\rho \sigma} \partial^{\mu} \overline{K^{0}} \\
-\mathcal{G}_{\mathrm{WZW}}^{\prime} \frac{N_{c} e}{12 \pi^{2} f_{\pi}^{3}} \frac{\sqrt{2}}{3} \epsilon_{\mu \nu \rho \sigma} A^{\sigma} \partial^{\mu} \overline{K^{0}}\left[\partial^{\nu} \pi^{-} \partial^{\rho} \pi^{+}\right] \\
+\mathcal{G}_{\mathrm{WZW}}^{\prime \prime}\left(\overline{K^{0}} \rightarrow \pi \pi \gamma \gamma\right)_{t e r m} .
\end{array}
$$

Note that the $\lambda_{+}$-insertion responsible for the first term in (24) is unique. For the other terms, however, it is not unique ; the $\lambda_{+}$can be inserted at several places within expression (19). Thus we focus on determining the coupling $\mathcal{G}_{\text {WZW }}$ from the uniquely parametrized $K^{0}$-decay into two photons - provided that this term in (24) explains the total $K_{L} \simeq K_{2} \rightarrow \gamma \gamma$ rate.

Neglecting CP-violation, we can determine $\mathcal{G}_{\mathrm{WZW}}$ from the measured $K_{2} \gamma \gamma$ coupling $C_{K_{2}}$ in eq.(9):

$$
\left|C_{K_{2}}\right|=\frac{2}{3}\left|\mathcal{G}_{\mathrm{WZW}}\right| C_{\pi^{0}} \quad ; \quad\left|\mathcal{G}_{\mathrm{WZW}}\right| \simeq 2 \times 10^{-7} .
$$

A sizable part of this coupling is reproduced by a quark-loop evaluation of Sect.2. It reflects a share of the anomaly contribution in the effective coupling $\mathcal{G}_{\text {WZw }}$, which comprises all possible short- and long-distance contributions, and as such has to be determined outside $\chi \mathrm{PT}$.

The second term in eq.(24) contributes to the decay $K_{L} \rightarrow \pi^{+} \pi^{-} \gamma$. Calculation within the low-energy QCD [5] shows that $\mathcal{G}_{\mathrm{WZW}}^{\prime}$ is suppressed by $M^{2} / \Lambda_{\chi}^{2}$ with respect to $\mathcal{G}_{\mathrm{WZW}}$.

\section{Discussion and Conclusions}

We have presented a new non-negligible contribution to $K_{L} \rightarrow \gamma \gamma$ obtained within the effective low-energy QCD from a short-distance operator. This contribution can account for about half of the experimental value, within the leading logarithmic approximation. Within the standard nomenclature [7] at the hadronic level, this process should belong to the class of reducible anomalous pole diagrams $K_{L} \rightarrow \pi^{0}, \eta \rightarrow \gamma \gamma$, which have so far been considered to be the only contribution of $\mathcal{O}\left(p^{4}\right)$. As already stated, this amplitude is subjected to pole cancellation, so that only some $\mathcal{O}\left(p^{6}\right)$ contribution should remain. The real representative of $\mathcal{O}\left(p^{4}\right)$ reducible anomalous neutral kaon decays remains

$$
K_{L} \rightarrow \pi^{+} \pi^{-} \gamma \gamma .
$$

How does our $\mathcal{O}\left(p^{4}\right)$ contribution to $K_{L} \rightarrow \gamma \gamma$ compare with the existing list of direct anomalous processes [7]? The origin of these direct anomalous terms 
can be traced back by following the authors of refs. [7, 8, 9]. They started from a four-quark effective hamiltonian essentially involving products of two weak currents (in the factorizable limit), using the functional derivative of the action $S=\int d^{4} x \mathcal{L}$ as an identification of the quark current:

$$
\bar{q} \gamma^{\mu} L q \sim \frac{\delta S}{\delta l_{\mu}}
$$

To obtain the anomalous terms, the authors of refs. [7, 8, 9] wrote down an expression of the form

$$
\mathcal{L}_{\text {An }} \sim G_{F}\left(\frac{\delta S^{(2)}}{\delta l_{\mu}}\right)\left(\frac{\delta S_{W Z W}}{\delta l^{\mu}}\right),
$$

where $S^{(2)}$ is the normal action $\mathcal{O}\left(p^{2}\right)$. The result did not contribute to $\overline{K^{0}} \rightarrow \gamma \gamma$ because a chiral-invariant expression typically contains (in the elecromagnetic case $\left.l_{\mu}=r_{\mu}=e Q A_{\mu}\right)$ the covariant derivative of $U, D_{\mu} U=$ $\partial_{\mu} U-i e A_{\mu}[Q, U]$, or $\mathrm{U}$ in the combination $U Q U^{\dagger}=Q+[\Pi, Q]+\ldots$. It is easily seen that the commutator $[\Pi, Q]$ does not contain neutral kaons $(Q$ acts as the unit matrix in the $s, d$ sector). To find a contribution giving the $\overline{K^{0}} \rightarrow \gamma \gamma$ amplitude in this way, one has to go further to $\mathcal{O}\left(p^{6}\right)$ terms. This should be no surprise when looking at the underlying quark processes. In the CP-conserving sector, where the CKM-favourable $s \rightarrow d \gamma \gamma$ has $u$ and $c$ quarks running in the loop, the SD contributions giving rise to $\mathcal{O}\left(p^{4}\right)$ terms are effectively cancelled by the GIM mechanism. Thus the neutral kaon decay representative of this direct anomalous class, which can be read off in refs. [0], 8], is

$$
K_{L} \rightarrow \pi^{+} \pi^{-} \gamma
$$

Our assertion is that our new WZW-extended $\Delta S=1$ term adds new "odd $\Pi$ " processes to the existing list of direct anomalous processes [7], namely the decay

$$
K_{L} \rightarrow \gamma \gamma
$$

We have arrived at additional a priori legitimate operators to the effective theory of the $\chi \mathrm{PT}$ type by integrating out the quark loops in an effective low-energy QCD. A guideline in introducing them are bosonized forms of the operators in the underlying quark theory. The new terms we have suggested rely on two-quark operators, which have not been considered as yet. Our contributions, termed "off-shell contributions" in ref. [2], were obtained in departing from the free-quark picture, in compliance with low-energy QCD. By scrutinizing the appearance of the anomaly in the U- and R-versions of low-energy QCD, we demonstrate both the viability of the anomaly-matching 
principle and the kinship of the $K_{L} \rightarrow \gamma \gamma$ and $\pi^{0} \rightarrow \gamma \gamma$ decays. The weak flavour transition, which distinguishes these two processes, is merely a decoration on the top of the dynamics underlying the anomaly. We hope that further investigation in this direction might enlighten the role of the chiral anomaly in flavour-changing radiative transitions and/or decode some secrets of bosonization in the Goldstone boson sector.

\section{Acknowledgement}

One of us (J.O.E.) wants to thank H. Bijnens, W. Bernreuther, R. Decker, G. Ecker, H. Neufeld and H. Pilkuhn for useful comments. I.P. acknowledges the hospitality of the Fysisk Institutt (Universitetet i Oslo), the partial support of the contracts CI1*-CT91-0893 (HSMU) and JF-899-31/NSF, and helpful discussions with H. Bijnens, G. Ecker and D. Klabučar.

\section{References}

[1] A. Pich and E. de Rafael, Nucl. Phys. B358 (1991) 311.

[2] J. O. Eeg and I. Picek, Phys. Lett B301 (1993) 423.

[3] J.O. Eeg, B. Nižić and I. Picek, Phys. Lett. B244 (1990) 513;

J.O. Eeg, B. Nižić and I. Picek, in Proceedings of the 25th International Conference on High Energy Physics, page 1259, Ed. K.K. Phua and Y. Yamaguchi, World Scientific 1991;

I. Picek, Nucl. Phys. B (Proc. Suppl.) 24A (1991) 101.

[4] G.J. Lin, J. Liu and Y.-P. Yao, Phys.Rev.Lett. 61 (1990) 1498;

H. Simma and D. Wyler, Nucl. Phys. B344 (1990) 283;

S. Herrlich and J. Kalinowski, Nucl. Phys. B381 (1992) 501.

[5] S. Weinberg, Physica 96A (1979) 327;

A. Manohar and H. Georgi, Nucl. Phys. B234 (1984) 189;

A. Manohar and G. Moore, Nucl. Phys. B243 (1984) 55;

S. Weinberg, Phys. Rev. Lett. 67 (1991) 3473;

J. Bijnens, H. Sonoda and M.B. Wise, Can.J.Phys. 64 (1986) 1;

D.I. Diakonov, V.Yu. Petrov and P.V. Pobylitsa, Nucl. Phys. B306 (1988) 809;

D. Espriu, E. de Rafael and J. Taron, Nucl. Phys. B345 (1990) 22;

J. Bijnens, Nucl. Phys. B367 (1991) 709;

A. Pich, CERN-TH 6368/92. 
[6] J. Wess and B. Zumino,Phys. Lett. B37 (1971) 95;

E. Witten, Nucl. Phys. B233 (1983) 422.

[7] G. Ecker, H. Neufeld and A. Pich, Phys. Lett B278 (1992) 337;

G. Ecker, H. Neufeld and A. Pich , CERN-TH.6920/93, June 1993.

[8] J. Bijnens, G. Ecker and A. Pich, Phys. Lett B286 (1992) 341.

[9] A. Pich,talk at the XXVIIth Rencontres de Moriond, CERN-TH.6511/92, May 1992.

[10] L.H. Sehgal and L. Wolfenstein, Phys. Rev. 162 (1967) 1362.

B.R. Martin and E. de Rafael, Nucl. Phys. B8 (1968) 131.

M.K. Gaillard and B.W. Lee, Phys.Rev. D10 (1974) 897.

E. Ma and A. Pramudita, Phys. Rev. D 24 (1981) 2476;

R. Decker, P. Pavlopoulos and G. Zoupanos, Z.Phys. C28 (1985) 117.

L.-L. Chau and H.-Y. Cheng, Phys. Rev. Lett. 54 (1985) 1768. ibid, B195 (1987) 275.

E. Golowich in "Flavour mixing and CP-violation", Ed. J. Tran Thanh Van, Edition Frontiers, 1985

J.F. Donoghue, B. Holstein and G. Valencia, Int. J. Mod. Phys. A2 (1987) 319;

J.F. Donoghue, B. Holstein and Y.C.R. Lin Nucl. Phys. B277 (1986) 651 ;

J.L. Goity, Z.Phys. C34 (1987) 341;

G. Ecker, A. Pich and E. de Rafael, Nucl. Phys. B303 (1988) 665.

A. Pramudita, Phys. Rev. D38 (1988) 3522.

[11] M. A. Shifman, A. I. Vainshtein, and V. I. Zakharov, Phys. Rev. D18 (1978) 2583;

Ya. I. Kogan and M. A. Shifman, Yad. Fiz. 38 (1983) 1454 [Sov. J. Nucl. Phys. 38 (1983) 628] .

[12] J.O. Eeg and I. Picek, Phys. Lett. B214 (1988) 651.

[13] J.O. Eeg and I. Picek, Phys. Lett. B224 (1989) 205.

[14] J.A. Cronin, Phys. Rev. 161 (1967) 1483. 